Article

\title{
Daily Variability in the Terrestrial UV Airglow
}

\author{
Thomas J. Immel ${ }^{1, *} \mathbb{C}^{\circ}$, Richard W. Eastes ${ }^{2}$, William E. McClintock ${ }^{2, *}$, Steven B. Mende ${ }^{1}{ }^{1}$, \\ Harald U. Frey ${ }^{1}\left(\mathbb{D}\right.$, Colin Triplett ${ }^{1}$ (D) and Scott L. England ${ }^{3}(\mathbb{D}$ \\ 1 Space Sciences Laboratory, University of California, Berkeley, CA 92521, USA; \\ mende@ssl.berkeley.edu (S.B.M.); hfrey@ssl.berkeley.edu (H.U.F.); triplett@berkeley.edu (C.T.) \\ 2 Laboratory for Atmospheric and Space Physics, University of Colorado, Boulder, CO 80309, USA; \\ richard.eastes@lasp.colorado.edu \\ 3 Department of Aerospace and Ocean Engineering, Virginia Polytechnic Institute and State University, \\ Blacksburg, VA 24061-0203, USA; englands@vt.edu \\ * Correspondence: immel@ssl.berkeley.edu (T.J.I.); bill.mcclintock@lasp.colorado.edu (W.E.M.); \\ Tel.: +1-510-643-3504 (T.J.I.)
}

Received: 12 June 2020; Accepted: 5 September 2020; Published: 30 September 2020

\begin{abstract}
New capability for observing conditions in the upper atmosphere comes with the implementation of global ultraviolet (UV) imaging from geosynchronous orbit. Observed by the NASA GOLD mission, the emissions of atomic oxygen (OI) and molecular nitrogen $\left(\mathrm{N}_{2}\right)$ in the 133-168-nm range can be used to characterize the behavior of these major constituents of the thermosphere. Observations in the ultraviolet from the first 200 days of 2019 indicate that the oxygen emission at $135.6 \mathrm{~nm}$ varies much differently than the broader Lyman-Birge-Hopfield (LBH) emission of $\mathrm{N}_{2}$. This is determined from monitoring the average instrument response from two roughly $1000 \mathrm{~km}^{2}$ areas, well separated from one another, at the same time of each day. Variations in the GOLD response to UV emissions in the monitored regions are determined, both in absolute terms and relative to a running 7-day average of GOLD measurements. We find that variations in $\mathrm{N}_{2}$ emissions in the two separate regions are significantly correlated, while oxygen emissions, observed in the same fixed geographic regions at the same universal time each day, exhibit a much lower correlation, and exhibit no correlation with the $\mathrm{N}_{2}$ emissions in the same regions. This indicates that oxygen densities in the airglow-originating altitude range of 150-200 km vary independently from the variations in nitrogen, which are so well correlated across the dayside to suggest a direct connection to variation in solar extreme-UV flux. The relation of the atomic oxygen variations to solar and geomagnetic activity is also shown to be low, suggesting the existence of a regional source that modifies the production of atomic oxygen in the thermosphere.
\end{abstract}

Keywords: airglow; thermosphere; atmospheric dynamics

\section{Introduction}

The research effort described here focuses on variability in the relative abundance of the two major constituents of the upper atmosphere, atomic oxygen (OI) and molecular nitrogen $\left(\mathrm{N}_{2}\right)$. It addresses this topic using remote spectrographically-separated, photometric measurements of the photo-emissions of the upper atmosphere from the NASA GOLD mission [1,2] that provides repeatable, daily measurements, with nearly identical observing geometry. Questions on the source of changes in these emissions and how they are related to changes in the underlying composition can be addressed with these new observations of the Earth's upper-atmospheric airglow. New techniques in separation of solar driven changes in brightness and those originating in changes in the atmosphere itself can now be implemented, as demonstrated here, in an effort to provide a more complete understanding of the behavior of the upper atmosphere and its response to varying inputs from above and below. 
Earth's upper atmosphere is the region above $\sim 100 \mathrm{~km}$ where densities drop to a level below which diffusion effectively promotes the separation of atmospheric species by mass with altitude. In this stratifying atmosphere, the ratio of light to heavy constituents (for instance, atoms of He vs. Ar) increases with altitude. Solar radiation in the 20 to $90-\mathrm{nm}$ range produces an ionized population of gas, including a super-thermal population of photoelectrons, while it dissociates molecular oxygen to the degree that atomic oxygen becomes the dominant atmospheric constituent above $200 \mathrm{~km}$, with $\mathrm{N}_{2}$ dominant at lower altitudes. Temperatures grow with increasing altitude because neither of these main constituents are efficient at radiating thermal energy, with temperature above $1000 \mathrm{~K}$ in the topside ionosphere during solar maximum (thus the term "thermosphere"). The temperature of the thermosphere directly affects the scale height of the separate constituents, and therefore the relative abundance of oxygen and nitrogen vs. altitude.

Temperatures change with solar cycle, from day to night, and in response to changes in radiative energetic processes in the lower thermosphere. The mix of OI and ionized species supports chemical processes that create new species including oxides of nitrogen that provide a cooling agent in the lower thermosphere, a population that can be enhanced by electron precipitation during major auroral events [3]. In interpreting data collected during storms occurring during periods of high solar activity, it is of compelling importance to characterize the behavior of the atmosphere during low activity. New observations from the NASA GOLD mission [1] provide many months of data during a period of very low solar activity, and are used in this study to investigate the quiet-time variability of Earth's ultraviolet emissions that provide key diagnostic measurements of conditions in the upper atmosphere.

\section{Observations of Earth's Ultraviolet Airglow}

The daytime thermosphere glows in ultraviolet light with three potentially bright sources. The first of these sources is resonantly scattered solar UV light that originates with solar atomic hydrogen and oxygen and is resonantly scattered by the same constituents in Earth's thermosphere in magnetic-dipole allowed transitions between accessible $2 p^{4}-2 p^{3} 3 s^{3}$ states in $\mathrm{O}$ and $1 s^{2}-2 p^{2}$ states in $\mathrm{H}$ [4]. The primary emissions of $\mathrm{HI}$ and $\mathrm{OI}$ are at $121.6 \mathrm{~nm}$ and $130.4 \mathrm{~nm}$, respectively, and are immediately recognizable as the two brightest features in spectrally separated UV light from Earth. The second major source is the fluorescence of thermospheric atoms and molecules, stimulated by the superthermal population of electrons originating in photionization and photodissociative ionization of thermospheric constituents $\mathrm{O}$ and $\mathrm{O}_{2}$. Photoelectron populations stimulate emissions of both $\mathrm{OI}$ and $\mathrm{N}_{2}$ that are also easily observed from space-based platforms. A third source is specifically an oxygen emission from the recombination of $\mathrm{O}^{+}$with ionospheric electrons, which is a minor contributor of the daytime OI emissions, but dominant at night. In this study of daytime UV emissions of the thermosphere, measures are taken to avoid contributions of ionospheric recombination emissions, so variations in emissions can be interpreted in terms of changes in the neutral atmosphere.

All of these sources contribute to the brightest 130.4-nm emission of OI, which has been a target of studies of the upper atmosphere and ionosphere since the 1960s, revealing variations in nighttime plasma density [5-7], daytime thermospheric composition [8-11], and the behavior of the polar aurorae [12-14]. The GOLD mission specifically avoids this emission, for the fact that the mix of sources complicates retrievals of thermospheric key parameters. Its focus is on emissions that in daytime are produced mainly by photoelectron stimulation.

Daytime photoelectron fluxes create significant ultraviolet emissions by atmospheric constituents in the 140-200 km altitude range, including the dominant constituent $\mathrm{N}_{2}$. Several atomic emissions so produced are dipole-forbidden and thus not readily produced by resonance of solar emissions, and are also therefore not readily scattered resonantly once produced in the upper atmosphere. This is the case for the atomic oxygen emission at $135.6 \mathrm{~nm}$, as well as molecular emissions that once emitted, are not readily reabsorbed or scattered. Emissions with strong local production that are not readily re-scattered are useful targets for remote sensing applications in that their brightness and altitude distribution 
often bear close relation to the properties of the region of the emission source. It is for this fact that recent NASA missions with UV imaging capabilities $[1,15]$ focus on the latter type of emissions.

The Global Observations of the Limb and Disk (GOLD) is one of these, a NASA mission of opportunity hosted on a communications satellite in a geostationary orbit parked over the mouth of the Amazon river [1]. From this vantage point, GOLD implements a scanning slit spectrometer to provide spectrally separated images of radiances in the ultraviolet spectrum from 133 to $168 \mathrm{~nm}$, collecting full disk images at these wavelengths with $\sim 30$-minute cadence in day and night by scanning the FOV defined by the slit across Earth's disk. The GOLD instrument has two slits that provide different spectral resolutions. In the daytime, the narrow slit is used to provide highest spectral resolution for retrieval of thermospheric rotational temperatures from the characteristic shape of several Lyman-Birge-Hopfield (LBH) emission bands of $\mathrm{N}_{2}$ [16]. Along with the LBH spectrum, another product is high resolution imaging of the emissions of atomic oxygen (OI) at $135.6 \mathrm{~nm}$, produced in the same altitude range as LBH emissions, largely through the same process of photoelectron impact fluorescence $[17,18]$. The instrument collects time-tagged events that indicate excitation of the detector by a UV photon, all of which are transmitted to the Science Data Center, where these data are binned to organize the data in three dimensions: two spatial dimensions and one spectral. Disk images are produced with 92 pixels in the east-west sense, and 104 in the north-south sense, each with 800 spectral bins. The thermosphere is optically thin to both of the emissions noted above, so they offer a source of diagnostic information on the conditions in near-Earth space related to their production, mainly the composition of the thermosphere in the same altitude range as the photoelectron peak. Numerous studies have demonstrated a particular capability to retrieve the ratio of $\mathrm{OI}$ and $\mathrm{N}_{2}$ from remote observations [19-22]. GOLD offers a remarkable new capability with an essentially stationary view of Earth, photon-counting detectors, and high spectral resolution allowing the exclusion of emissions that reduce the accuracy or precision of the retrieval algorithms.

The aspect of the GOLD observations that is the focus of this study is the change in the airglow brightness with time. This question originates from evaluation of summary views of the ratio of OI to $\mathrm{N}_{2}$ in the thermosphere retrieved by GOLD. This is related almost linearly to the ratio of 135.6-nm to LBH emissions of the thermosphere [19]. An ongoing debate over the effect of temperature on this relation is unresolved; where Zhang et al. [23,24] recently argued that a solar flux related variation in $\mathrm{O} / \mathrm{N}_{2}$ is related to the change in temperature in the lower thermosphere, [25] argue that the emission ratio that provides the $\mathrm{O} / \mathrm{N}_{2}$ product is independent of the thermospheric temperature. The question of the extent to which the ratio of these emissions is related to temperature can be addressed by surveying the changes of the two emissions over time, under similar conditions of solar illumination, latitude and longitude, and viewing angle, and possibly including the temperature product that GOLD provides [2]. The results presented here offer insight into the behavior of these emissions at solar minimum, and potential variations in the $\mathrm{O} / \mathrm{N}_{2}$ product that originate in processes not yet considered.

\section{Results}

As noted above, the GOLD hyperspectral imager offers simultaneous observations of thermospheric oxygen and nitrogen emissions, and we utilize the Level 1 daytime product: geolocated raw instrument counts, reported for UV wavelengths from 134 to $168 \mathrm{~nm}$. These are obtained using the instrument in its daytime, high spectral resolution mode (narrow entrance slit), and so provide signatures of several key features of Earth's airglow, and allow for clear separation of the emissions of atomic oxygen and atomic and molecular nitrogen. Here we use version 2, revision 1 of the product, downloaded from the NASA Space Physics Data Facility. An example of the spectrum that can be retrieved is shown in Figure 1 where the signal in 25 neighboring pixels is co-added to provide an average example spectrum representative of counts collected over a single sweep of the slit across the dayside. Raw counts are single events in the event-counting detector, and are used for this study to provide a clear basis for statistical interpretation. The detector is a linear system, and detector event rates can also be interpreted in terms of absolute brightness of the remotely viewed target, to the limits of the 
accuracy of the available calibration. That said, an absolute calibration is not necessary for this study. The relative counting rates of different emissions in any pixel is the focus of this work, and as will be made clear: all that is required to evaluate the variations in brightness of the 135.6 and LBH emission is that the the responsivity of the instrument not vary by more than the root-mean-square (RMS) of the instrument counts measured in the analysis boxes over periods shorter than a week. As we will show, working with count rates of $\sim 1000$ events per pixel and the number of pixels in each analysis region of $\sim 125$ would imply instrument requirement that its responsivity not change more than $1 \%$ per week. Given the design requirements of the GOLD mission and its on-orbit performance [26], this requirement is easily met with the current data.

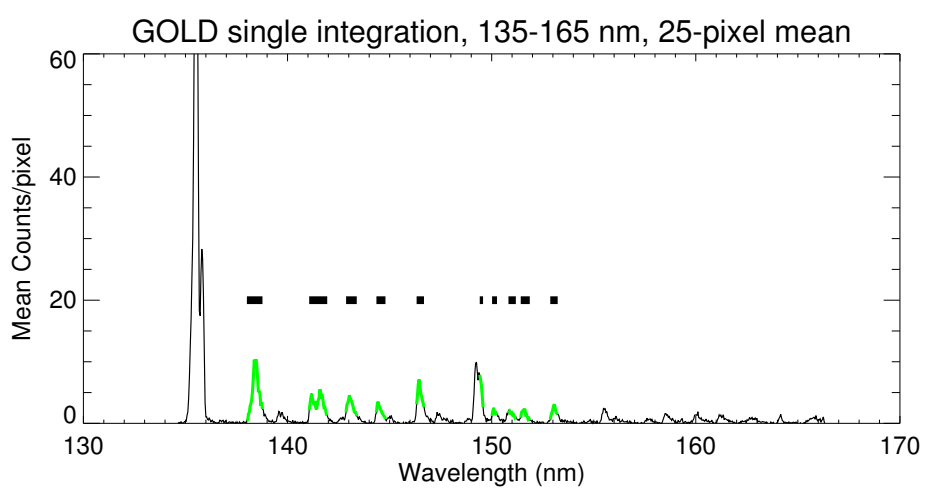

Figure 1. An average GOLD spectrum for a $5 \times 5$ batch of pixels from one image on day 15 of 2019 at 06:10 UT. The full spectrum is shown and the portions extracted to construct LBH (Lyman-Birge-Hopfield) images. The bright feature at $135 \mathrm{~nm}$ is the $2 \mathrm{p}^{4}{ }^{3} \mathrm{P}-2 \mathrm{p}^{3} 3 \mathrm{~s}^{5} \mathrm{~S}$ oxygen doublet (resolved by GOLD) combined with an LBH band, shown in detail in the next figure. Portions of the spectrum that are extracted from each pixel are underscored with a black horizontal bar and also noted in the mean counts line with a green color.

\subsection{Isolation of 135.6-nm and LBH Emissions}

An example of the response of GOLD to UV observations of Earth is shown in Figure 1. The two primary features in Figure 1 are the detector's response to the OI doublet emission near $135.6 \mathrm{~nm}$ that produces dozens of counts $/ \mathrm{nm} /$ pixel, and the broadly distributed band emission of $\mathrm{N}_{2}$ at longer wavelengths. These emissions identified and used here as LBH counts are marked with a overbar in the figure and highlighted with green traces, which are co-added to produce a reference LBH-per pixel image. The bright NI emission at $149.3 \mathrm{~nm}$ is specifically excluded from this addition, as well as the 135.6-nm emission of OI, and some of the more dim LBH emissions. In all, the counts from 123 wavelength positions are co-added to provide total LBH counts for each pixel in the GOLD images.

The oxygen feature itself is in close proximity to a LBH-band emission feature at $135.4 \mathrm{~nm}$. While not completely separated from the 135.6-nm emission of OI, the GOLD instrument has the spectral resolution to characterize the effect of this emission on the profile of 135.6-nm emission and, therefore, allow for the exclusion of this contaminant from a characterization of the 135.6-nm emission. The profile of summed GOLD counts around $135.6 \mathrm{~nm}$ is shown in Figure 2. Beneath the profile, the central locations of the LBH band, and the two components of the 135.6 doublet are identified with vertical lines, which are of sizes roughly proportional to the expected relative brightness of the specific emissions [4,27], with approximately $200 \mathrm{R}$ in the LBH band at $135.4 \mathrm{~nm}$, and approximately $1 \mathrm{kR}$ in the total 135.6-nm emission, in a 3:1 proportion for temperatures near $500 \mathrm{~K}$ at the expected altitude range of 145-155 km. From the GOLD instrument, only counts from a wavelength range where the trace is colored red and marked with an overbar are considered as contributors to the 135.6-nm emission, co-adding counts from 15 wavelength positions to provide total OI 135.6 counts for each pixel in the GOLD images. 


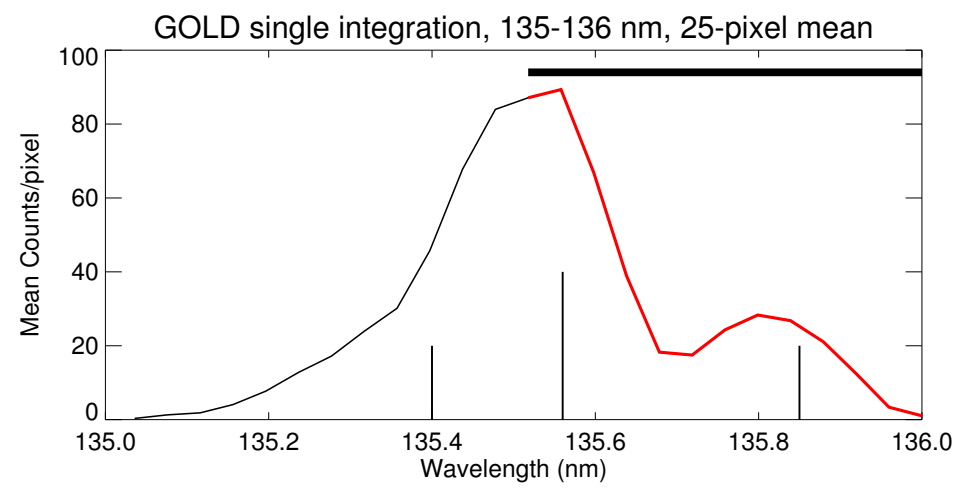

Figure 2. An average GOLD line profile at the 135.6 emission feature for a $5 \times 5$ batch of pixels from one image on day 15 of 2019 at 06:10 UT. The doublet of OI is evident, with 2 vertical lines indicating the theoretical emission value. An LBH band at 135.4 is also part of this feature, and so for further use in this report, only counts in the wavelength regions indicated with a red count line, and marked with a heavy overbar, are used to represent OI 135.6 emissions.

Using the described steps, the LBH and 135.6 emissions are effectively isolated, providing the means to process the data to provide separate images. This is done to inform the retrieval of column density ratio of oxygen to nitrogen [28], a baseline GOLD data product. For this work, we assess the instrument response to these two emission separately. Examples of the images that are produced for this study are shown in Figure 3, using data from Channel 1 of the instrument obtained around 15:00 UT on 21 January 2019. Here we explain how these are compiled.
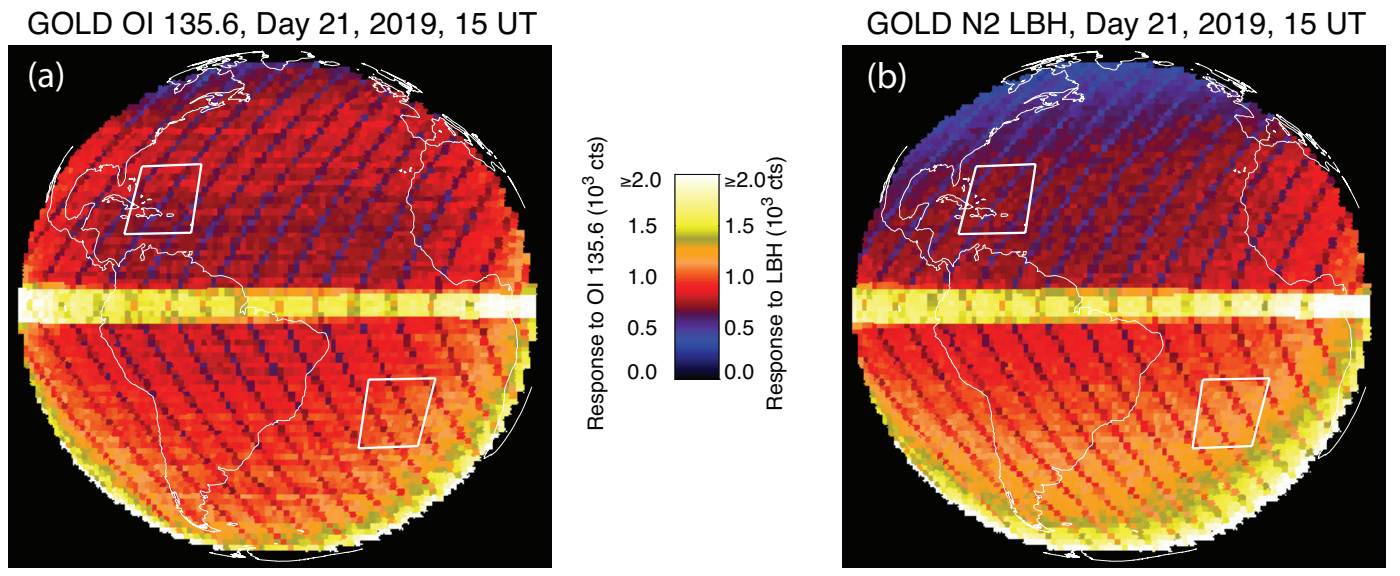

Figure 3. Images of the response of the GOLD instrument to 135.6 (a) and LBH (b) airglow, indicating airglow analysis boxes. The images are made by combining the 4 instrument scans between 15 and 16 UT, providing a global view. Discontinuities at the equator are an artifact of overlapping scans where analysis is avoided. Regular pattern variation is the result of variations in integration times at the time of rebinning of instrument counts to form images, is identically represented in every image from GOLD. The locations of the analysis boxes are selected to sample both hemispheres and minimize contamination of the $135.6-\mathrm{nm}$ emission by ionospheric $\mathrm{O}^{+}$recombination.

\subsection{6-nm and LBH Images}

In the daytime airglow operating mode, the GOLD instrument sweeps its FOV across the northern and southern hemispheres in successive passes. During a sweep, each photon-event that excites the detector is recorded, producing a time-tagged list of event positions on the detector. With the entrance slit defining a tall, narrow FOV, and a UV grating dispersing the light, the location of an event on the detector reflects the entrance angle of the photon and its wavelength [26]. Reconstruction of an image obtained during a scan therefore requires knowledge of both the position of the event on the 
detector and the position of the scanning FOV at the time of the event. Relying on the GOLD project's processing, we select for use Version 2 of the Level 1C Channel A Daytime product provided by the GOLD Science Data Center that provides raw instrument counts with 94 samples in the scanning direction, and 104 samples along the slit (vertically). There are 800 wavelength bins for each pixel. This vertical sampling covers the full north-south range of the instrument, filled with the measured total raw counts from the top down to the equator, and from the bottom up to the equator in alternating scans. To produce complete global images for this study, data from four consecutive sweeps of the FOV are co-added. This provides full global imaging, with overlap at the equator. The total number of raw counts in the wavelength regions described in the previous section, where the scans provide two samples for pixels over most of Earth's disk, and four samples in the region where the scans overlap. The mapped image is shown from the perspective of the GOLD observatory at its vantage point in the geostationary Clarke orbit that its host communications satellite achieved [26]. It represents a co-addition of raw counts from three scans obtained prior to $15 \mathrm{UT}$ and the first obtained after $15 \mathrm{UT}$. On this date, the images have timestamps at 14:22, 14:40, 14:52, and 15:10 UT, collecting data over $60 \mathrm{~min}$. For this study, these data are consistently obtained at the same times from one day to the next.

The analysis of airglow focuses on the day-to-day changes in the intensities of the two emissions. These changes are evident in a cursory examination of images of the ratio of the two emissions over time, as structures appear and change from one day to the next [29]. This study focuses first on the independent brightness variations in each emission. The brightness varies across the globe with changes in solar illumination and with the viewing angle, both of which can be characterized by zenith angle. The prior effect is due to solar flux variations relative to the local horizontal plane, the latter due to changes in the length of the integrated column of airglow emissions along the viewing line of sight $[11,19]$. To minimize the effect of these factors in this analysis, we select fixed regions of the Earth to track, the pixels always at the same viewing angle from GOLD, and as noted above, using data obtained around 15 UT each day, near the time of local noon at the GOLD sub-satellite point. Because the integration times repeat consistently from one day to the next, the solar local times vary only with the synodic variation over a year. The remaining variation in solar zenith angles is slow, with season, introducing a variation at a frequency far outside of the day-to-day effects investigated here.

The images in Figure 3, show the total raw counts per pixel. Small differences in the per-pixel integration time used in building images from time-tagged photon events appear as diagonal, herringbone strips. While the observations are nearly continuous in latitude and longitude $(<50 \mathrm{~km})$, they have been binned to the nearest pixel center in the grid (a constant angular grid that is $125 \mathrm{~km} \times 125 \mathrm{~km}$ at nadir), and the per-pixel integration time is the same for LBH or OI emissions. Another artifact stands out, the horizontal track of $2 \times$ counts where the FOVs overlap. Since the analysis uses data from the regions marked by white boxes (discussed shortly), areas in this region are excluded, though they are regularly used in deriving higher level products. While calculation of radiances [2] remove all these patterns, the effect of those corrections on the statistical uncertainties could complicate the analysis we perform, so we work only with raw instrument counts. All the photon events produced in the imaging period are represented here, and the number of data samples included in each pixel was consistent (for each latitude and longitude bin in the fixed grid) during the study period. The total integration time in the data sample from the two geographic regions selected for analysis (in the next section) and the number of pixels (125) remains exactly the same for the 200 days of this study. We can proceed with confidence that all the photon events observed by GOLD are used in this analysis.

We select the first 200 days of 2019 for analysis. The data from the aforementioned universal times are used every day to evaluate the brightness of the two airglow signatures in successive views separated by exactly $24 \mathrm{~h}$. This provides a consistent baseline with the only variation coming from the slow synodic offset between UT and solar local time, and the slow seasonal variations in solar angle. 


\subsection{Airglow Variations in 2019}

To evaluate thermospheric airglow signatures, it is important to consider the fact that the ionospheric recombination emissions are present in the 135.6-nm channel, a product of $\mathrm{O}^{+}$recombining with $\mathrm{e}^{-}$. This well-known signal is a GOLD product in the nighttime, where photoelectron fluxes disappear along with LBH emissions, and the only remaining source in the GOLD passband is recombination 135.6-nm photons [30], and an even more minor $\mathrm{O}^{+}-\mathrm{O}^{-}$mutual neutralization reaction that constitutes $\sim 10 \%$ of the ionospheric source [31]. In this analysis focused on atmospheric sources, we take steps to minimize these ionospheric sources. To do so two analysis regions in the GOLD FOV, are located at middle latitudes, away from the geomagnetic equator and equatorial ionospheric anomalies while also avoiding regions of potential auroral contamination. The regions selected are shown in Figure 3 over the Caribbean sector, and the South Atlantic. Each extends 15 degrees in both latitude and longitude, one centered at 22 North; the other at 22 South. They are placed 25 degrees in longitude from the approximate GOLD longitude of $47 \mathrm{~W}$.

Having taken steps to minimize the contribution of recombination emissions, we still must consider the remaining signal of the ionosphere. Per the research effort undertaken to prepare for thermospheric constituent retrievals for the NASA ICON mission [32], simulations of the daytime 135.6-nm emission predict that the relative brightness of the ionospheric recombination components is approximately $2-3 \%$ of the total daytime $135.6-\mathrm{nm}$ brightness for a peak ionospheric density $(\mathrm{NmF} 2)$ of $\sim 10^{6}$ electrons $/ \mathrm{cm}^{2}$ [33]. This value of $\mathrm{NmF} 2$ is generally higher than what is observed at middle latitudes, particularly under the low solar EUV flux conditions of 2019, and certainly in the morning sector where the F-peak is recovering from nighttime decay. Furthermore, while the photo-electron produced emissions observed by GOLD are also low due to solar minimum conditions, those will drop in proportion to the reduction in solar flux, while ionospheric recombination emissions will drop as the square of the F-region electron densities. That is, ionospheric recombination emissions are likely present in very low proportion to photoelectron-produced emissions. We find therefore that the assumption of an ionospheric contribution of $2-3 \%$ to the total 135.6-nm signal is a conservative estimate and is unlikely to be any higher. Given the period of low geomagnetic activity in early 2019, and the lack of auroral disturbance effects for most of this period that could modify the ionosphere, the worst case NmF2-related variations are at most $25 \%$ from day-to-day. Ionospheric emissions should therefore not introduce a variation of more than about $1 \%$ in the response of the GOLD instrument to 135.6-nm emissions in these regions, a value similar to what is required of the instrument sensitivity performance discussed at the start of the Results section.

The mean observed raw counts are determined by calculating the average of the observed counts per pixel for the pixels in these regions. These boxes provide $\sim 125$ pixels for analysis, reducing the expected statistical RMS of the mean counting rate in the respective analysis boxes by a factor of approximately 11 . These mean values are calculated for every 15 UT combined image, where the combination of two scans further reduces the expected per pixel mean RMS by another factor of $\sqrt{2}$ (the analysis locations are outside of the region of overlap where there four scans contribute to the total counts). The mean counts and corresponding standard deviations in each region, for each wavelength band, are used throughout the following analysis, which is summarized Figure 4 and discussed next. Other key information such as the geomagnetic Ap index [34], and the solar $10.7 \mathrm{~cm}$ radio flux (F10.7) [35], both of which have been associated with changes in Earth's UV emissions are also shown. In the case of Ap, the expected effect is in a relative sense, by potentially modifying the ratio of OI vs. $\mathrm{N}_{2}$, and in the case of F10.7, a proxy for solar EUV emissions that drive photoelectron production in Earth's thermosphere, the brightness of the UV wavelengths studied here may likely vary together with the index.

The mean counting rates measured in the two regions of interest for both emissions over the 200 day period are shown in the top two panels of Figure 4, with the results from the regions shown in black (northern/morning) and red (southern/afternoon), referred to as the NH and SH regions, respectively. The upper left panel shows the response to 135.6-nm emissions, the upper right shows 
the same for LBH. Each daily mean value is shown as a vertical bar indicating the range of uncertainty in the mean value, based upon the statistical analysis of the distribution of values measured in the $\sim 125$ individual pixels included in the geographically extended sample (as shown in Figure 3). The standard deviation of the mean varies around 8-10 mean counts per pixel. Two sets of data are shown for each analysis box, the day-to-day data just described and a running 7-day average, using the same color and a solid line. It is clearly evident when the day-to-day mean values depart from the running weekly mean. The mean response per pixel to 135.6-nm varies around 780 and 870 counts in the $\mathrm{NH}$ and $\mathrm{SH}$, respectively (averaged over the entire period), with an overall standard deviation of 28 and 23 mean counts per pixel. Instrument response to LBH is slightly higher with the mean response per pixel over the period of 900 and 970 , respectively in the $\mathrm{NH}$ and $\mathrm{SH}$, with a standard deviation around 30 mean counts per pixel in each case.
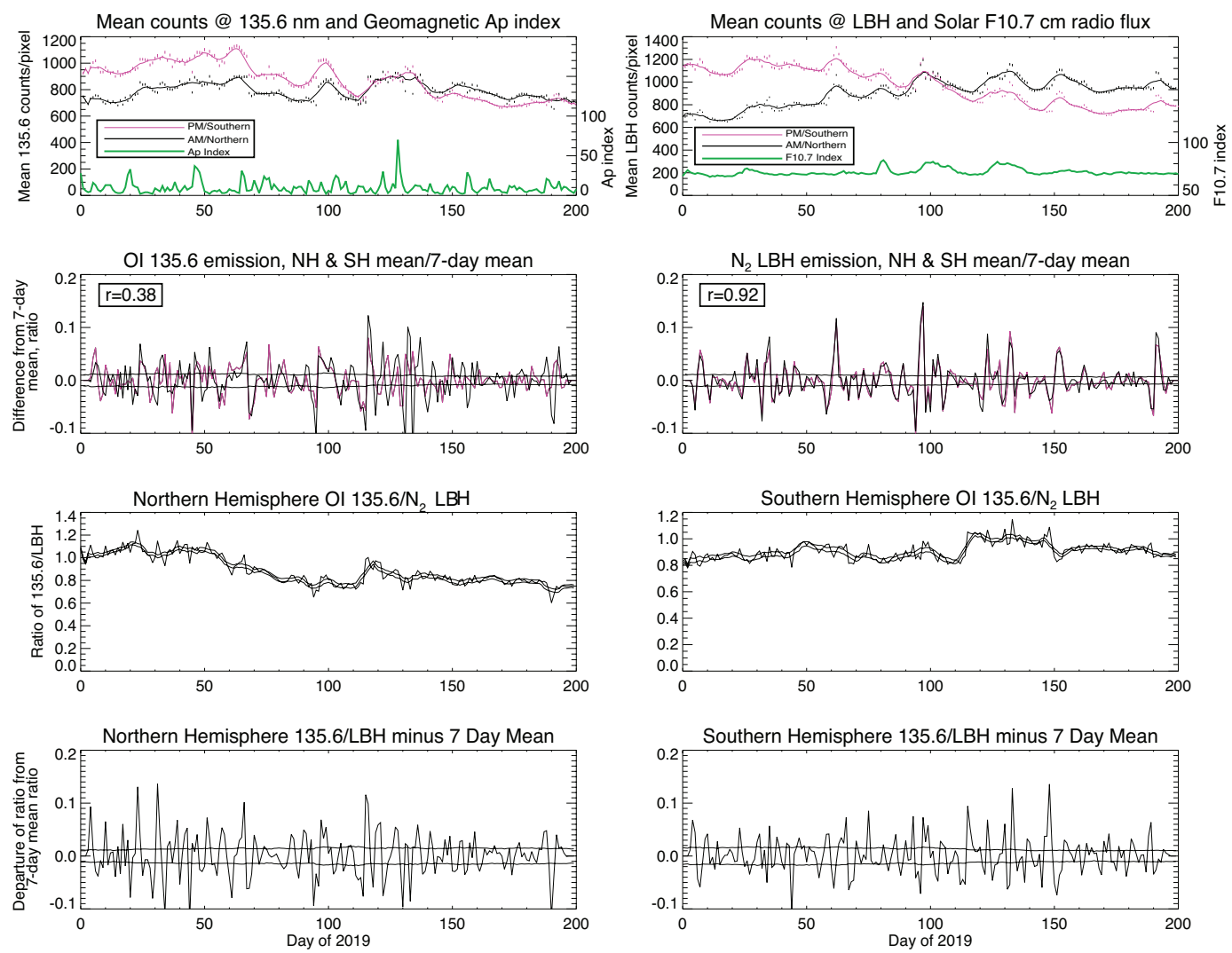

Figure 4. The mean GOLD instrument response to 135.6 and LBH airglow in the analysis boxes indicated in Figure 3 is shown over 200 days at 15 UT (12 Local Time at the satellite) in the top row. Shown for reference are proxies for geomagnetic activity (Ap index), and Solar radiance (10.7 cm flux). The second row shows the departures of OI 135.6 and $\mathrm{N}_{2} \mathrm{LBH}$ from the seven day average in both hemispheres, as a ratio to the average. Reported here are the correlation coefficients of the $\mathrm{NH}$ and SH data for each emission. The third row shows the ratio of $\mathrm{OI}$ and $\mathrm{N}_{2}$ counts in the separate hemispheres. The fourth row shows the departure of this ratio from the 7-day mean. The standard deviation from the mean values is calculated and shown with the height of the data point in the top row, and with horizontal lines in the last 3 rows.

The two potentially influential parameters discussed above are shown; the daily geomagnetic Ap index (green line, upper left), and F10.7 (green line, upper right). Regarding these two potential influences, the variations over time of all the emissions show features mainly related to the variation in the F10.7 flux. In regard to the level of geomagnetic activity, it can be characterized as low throughout the period, with highest activity on day 129. At low-to-middle latitudes, this can generally introduce an increase in oxygen densities, as any related heating in the auroral zones produces upwelling of $\mathrm{N}_{2}$ 
and a general equatorward migration of lighter constituents like OI [36-38]. If this effect is observed here, it is an abrupt drop in the NH OI 135.6 counts, seen in the day-to-day day mean values, while for several days after the event the mean OI 135.6 counts in the $\mathrm{SH}$ region remain well above the 7-day mean. Additionally, the mean LBH counts in both regions also rest well above the 7-day mean for the two days following the sharp increase in Ap. These variations reflect the natural variability that will continually be present in these data, as seen here during solar minimum conditions.

Beyond these changes due to geomagnetic and solar influences, there are other remarkable behaviors to note in these data. First, the trend in either the OI or $\mathrm{N}_{2}$ emissions in the respective northern and southern hemispheres shows a similar behavior over time. Where the southern hemisphere is brighter in each emission at the start of the year, there is a notable reversal in the relative brightness between the hemispheres around day 100, where the northern hemisphere is generally brighter afterwards. This is mainly due to the change in solar zenith angles, where around day 85 the sub-solar point crosses the equator and continues north. The OI and $\mathrm{N}_{2}$ emission vary differently, and this is likely a seasonal effect, where together the middle latitudes are transitioning from a solstice condition to an equinoctial condition $[39,40]$. Overall, the instrument responses vary in an expected manner, providing confirmation that the GOLD data reveal basic geophysical/geometric variations. These are essentially annual variations that do not interfere with an analysis of the day-to-day variability.

Closer examination of these values is made possible through examination of the differences between the mean observed counts and the 7-day mean. This effectively eliminates variations on longer-than weekly time scales, shown as a ratio in the second row of Figure 4 , where ratio $=$ (mean counts -7 -day mean)/7-day mean. The colors indicate $\mathrm{NH}$ or $\mathrm{SH}$ as in the first row. The standard deviation of the individual mean values divided by the 7-day average is shown for reference, above and below the ratio $=0$ level, and is at the $\sim 1 \%$ level. For the purpose of a simple representation, only the SH bars are shown, valid because the $\mathrm{NH}$ bars are of similar magnitude. As is already evident from the first row, several days in each time series depart from the 7-day mean in ways that cannot be attributed to counting statistics, with an RMS variation 2.5-3.5 larger than can be attributed to statistical uncertainty in the calculated mean values. One can further evaluate the brightness changes together, and look for a correlation between the instrument response to OI and LBH emissions in the two regions. Comparing the OI 135.6-nm emissions between the NH and SH regions, the Pearson correlation coefficient is calculated. For OI emissions the correlation between these data is 0.38 , while for a similar treatment of the $\mathrm{N}_{2}$ emissions, the correlation is much higher at 0.92 .

This reveals a second remarkable behavior in the airglow variations overall. In two independent samples on the dayside, the LBH emissions vary in remarkably similar fashion, while the OI emissions show more uncorrelated behavior. The high correlation implies a common source to these changes in LBH, where variations in solar inputs offer the most direct explanation. Though the $5-10 \%$ changes are large for solar minimum, the largest outliers occur on days that roughly correspond to the small enhancements in F10.7 index, possibly indicating the occurrence of solar flares during these periods, that would be linked to changes in solar EUV flux and enhanced photoelectron production in the thermosphere. This should obviously have concomitant effects on the production of both LBH and OI 135.6 emissions. The question then is clearly why do the OI 135.6 emissions in the $\mathrm{NH}$ and $\mathrm{SH}$ regions show a much lower correlation that the LBH emissions? It could be that the OI densities vary more significantly due to geophysical effects. Furthermore, given their different scale heights and excitation cross-sections, OI 135.6 may be generated at a different height than LBH, where different physical processes may be present. Further investigation is warranted, an area covered in the next section of this paper.

Comparison of the mean counts of 135.6 and $\mathrm{LBH}$ from the same $\mathrm{NH}$ and $\mathrm{SH}$ regions shows another notable result. A brief analysis shows that the variations of the two emissions are poorly correlated, with correlation coefficients of 0.08 and 0.11 in the $\mathrm{NH}$ and $\mathrm{SH}$, respectively. Both are expected to be influenced by solar radiation, so this correlation is remarkably low. This is evidence of significant changes in OI abundance that are not matched by changes in the $\mathrm{N}_{2}$ abundance, though, 
as above, consideration of the altitude distribution of the emissions is warranted to support any conclusion in this regard.

To evaluate the potential variations in OI relative to $\mathrm{N}_{2}$, the ratio of the two instrument counting rates is investigated in both $\mathrm{NH}$ and $\mathrm{SH}$ regions, first in a direct calculation, then in terms relative to the 7-day mean of their ratio. The ratio of OI $135.6 \mathrm{vs}$. $\mathrm{N}_{2} \mathrm{LBH}$ mean counts is shown in the third row of Figure 4 separately in the $\mathrm{NH}$ and $\mathrm{SH}$. Error bars that combine the uncertainty of the daily mean of each source are overlain for reference. This ratio can be viewed as a proxy for the ratio of OI to $\mathrm{N}_{2}$ in the thermospheric column, given that abundances in the photoelectron-excited region reflect the relative abundances of the constituents at lower and higher altitudes [19]. Further analysis is required to accurately retrieve that product [2], but the trends in this ratio allow for the interpretation of changes in the relative abundance of $\mathrm{OI}$ and $\mathrm{N}_{2}$ over the study period. Here, the trend in the $\mathrm{NH}$ region toward lower OI vs. $\mathrm{N}_{2}$ is evident, corresponding to the slow change in the thermosphere from wintertime high OI abundance to lower abundance generally observed in summertime. Though the SH does not exhibit such a significant change from its summertime condition, a longer time series would likely reveal this change.

The last row of Figure 4 shows the deviations of the ratio of OI 135.6 vs. $\mathrm{N}_{2} \mathrm{LBH}$ from the 7-day mean of the values in the $\mathrm{NH}$ and $\mathrm{SH}$. Here we find that the ratio changes with an RMS of about $3-4 \%$ from one day to the next, with larger deviations occurring on several days in both regions on the order of $10 \%$. It is not evident that any of these large departures clearly match any of the periods of increased geomagnetic activity indicated by the Ap index (top row).

\section{Discussion}

The open question that should be addressed is whether the indications of different behavior of the OI and $\mathrm{N}_{2}$ emissions are related to modification of the photoelectron flux, or changes in the relative density of the thermospheric constituents? In either case, is it due to waves that are caught in the act of modifying the region, or is it a day-to-day change in the properties of the atmosphere well below that are responsible, introducing changes that are then balanced as the thermosphere maintains diffusive equilibrium? On the other hand, is the larger variation in OI emissions due to the fact that the atomic oxygen population is excited by solar radiance variations that do not affect $\mathrm{N}_{2}$ in the same way?

Without measurements of the system beyond the UV disk radiances, or a set of physical simulations of this 200-day period, it is difficult to ascribe the variations in relative brightness to any particular phenomenon. Such measurements of the daytime thermosphere are now available with new observations from NASA's ICON mission $[15,32]$ that carries capability for measuring temperatures, winds and atmospheric waves in the thermosphere. This mission carries a high-spatial resolution FUV spectrographic imager [41] that provides limb observations at middle latitudes that complement the disk observations of GOLD, and in fact can be used in this study to provide more information on the source of the emissions. The ICON FUV spectrographic imager views the limb up to $\sim 450 \mathrm{~km}$ altitude and portions of the disk under the limb at two wavelengths centered at 135.6 and $157 \mathrm{~nm}$, measuring OI and LBH emission profiles. From the ICON orbit, it can sample morning and afternoon airglow at middle latitudes for comparison to GOLD's measurements.

The specific question ICON can address is where the emissions of OI and LBH are coming from in the thermosphere. Because the thermosphere is optically thin at these wavelengths, ICON's limb observations can pinpoint the altitude of the emission peak and inform discussion of the source of the UV variations. Modeling results by Stephan et al. [33] predict that the peak in the volume emission rate of the 135.6 emissions is higher by about $20 \mathrm{~km}$ than the $\mathrm{LBH}$ emission. This is due primarily to the fact that the thermospheric $\mathrm{N}_{2}$ density profile drops much more significantly with altitude than OI, even as the EUV-produced photoelectron flux that excites the emissions is identical. A $20 \mathrm{~km}$ separation is approximately the scale height of $\mathrm{N}_{2}$ and half the scale height of $\mathrm{OI}$, so it is possible to consider that some process affecting the LBH layer may change with altitude and affect the 135.6-nm layer in a more pronounced manner. Examples of this kind of process are upward propagating gravity 
and acoustic waves that have been modeled and observed in the thermosphere [42,43], and recently observed in Earth's 135.6-nm emissions by GOLD [44].

An observation of the tangent height profiles of the LBH and 135.6-nm airglow is shown in Figure 5. These come from ICON observations on days 338 and 355 of 2019, from recently commissioned instrument data. These are mean altitude profiles of the calibrated LBH and 135.6-nm emission intensities in Rayleighs from a combined set of 80(30) profiles measured at $\sim 30 \pm 5^{\circ}$ latitude in the $\mathrm{AM}(\mathrm{PM})$ sector. Access to the AM and PM sectors is gained on separate days as the ICON orbit precesses in local time. No inversion to retrieve volume emission rate is performed; the goal is to identify the observed altitude of the emission peak. The mean emission profiles are shown for LBH using individual points, and the brighter 135.6-nm emission is shown with a solid black line. In each sector, the peak in these emissions is around $150 \mathrm{~km}$. The actual values are tabulated below in Table 1, where the altitude resolution afforded by the instrument is $\sim 5 \mathrm{~km}$ :

Table 1. Peak altitude of OI 135.6 and $\mathrm{N}_{2}$ LBH emissions.

\begin{tabular}{lcc}
\hline & AM & PM \\
\hline 135.6 Peak Altitude & $153 \mathrm{~km}$ & $153 \mathrm{~km}$ \\
LBH Peak Altitude & $146 \mathrm{~km}$ & $147 \mathrm{~km}$ \\
\hline
\end{tabular}

It is evident that the altitude separation in the emissions is small compared to the scale height of either constituent, and that the peaks are broad with intensities greater than half the peak emission extending dozens of kilometers above it in every case. Though the altitude profile of the volume emission rate is not retrieved (it would fall to zero below $100 \mathrm{~km}$ ), the peak and topside profile are indicators of similar emission profiles, and as such, processes affecting the neutral densities should be influencing both constituents at the same altitude. Considering any physical process that is driving day-to-day variations in brightness, no argument can be made that the growth or diminution of this process with altitude could be the cause of the difference in the behavior of the emissions. We therefore posit that variation of the OI densities themselves, within the region of high photoelectron flux, are the cause of the brightness variations in OI 135.6.

Given the earlier finding that, given their simultaneous global variation, the $\mathrm{N}_{2} \mathrm{LBH}$ variations are likely attributable to short-term solar variation, what follows naturally is the finding that the variability in OI 135.6 emissions seen in Figure 4 is evidence that the density of OI varies to a greater degree in the lower thermosphere than that of $\mathrm{N}_{2}$. The day-to-day changes could be indicative of large temperature changes at the base of the thermosphere, that cause changes in the profiles of both OI and $\mathrm{N}_{2}$, with a larger relative effect on the lighter constituent. These could be tied to regional scale variations in non-migrating tides, propagating upward from the troposphere and stratosphere [45], which may exhibit their own day-to-day variability $[46,47]$. However, our finding that the LBH and OI variations are poorly correlated does not support an explanation along these lines.

Consideration of the unique nature of atomic oxygen, that unlike $\mathrm{N}_{2}$ it is an ephemeral and reactive constituent of the upper atmosphere, is clearly due. The processes of its production and loss involve a number of chemical reactions that occur in the thermosphere and mesosphere $[48,49]$. Though the energy balance of the mesosphere-lower-thermosphere system under these conditions varies slowly under these low solar activity conditions, it is possible that the dynamical environment of the region, dominated by tidal and planetary wave forcing originating in the troposphere and stratosphere, drives additional variability that modifies OI densities above, which is elucidated by comparison of the thermospheric 135.6-nm emission relative to the more stable LBH emissions, as performed in the course of this research. 


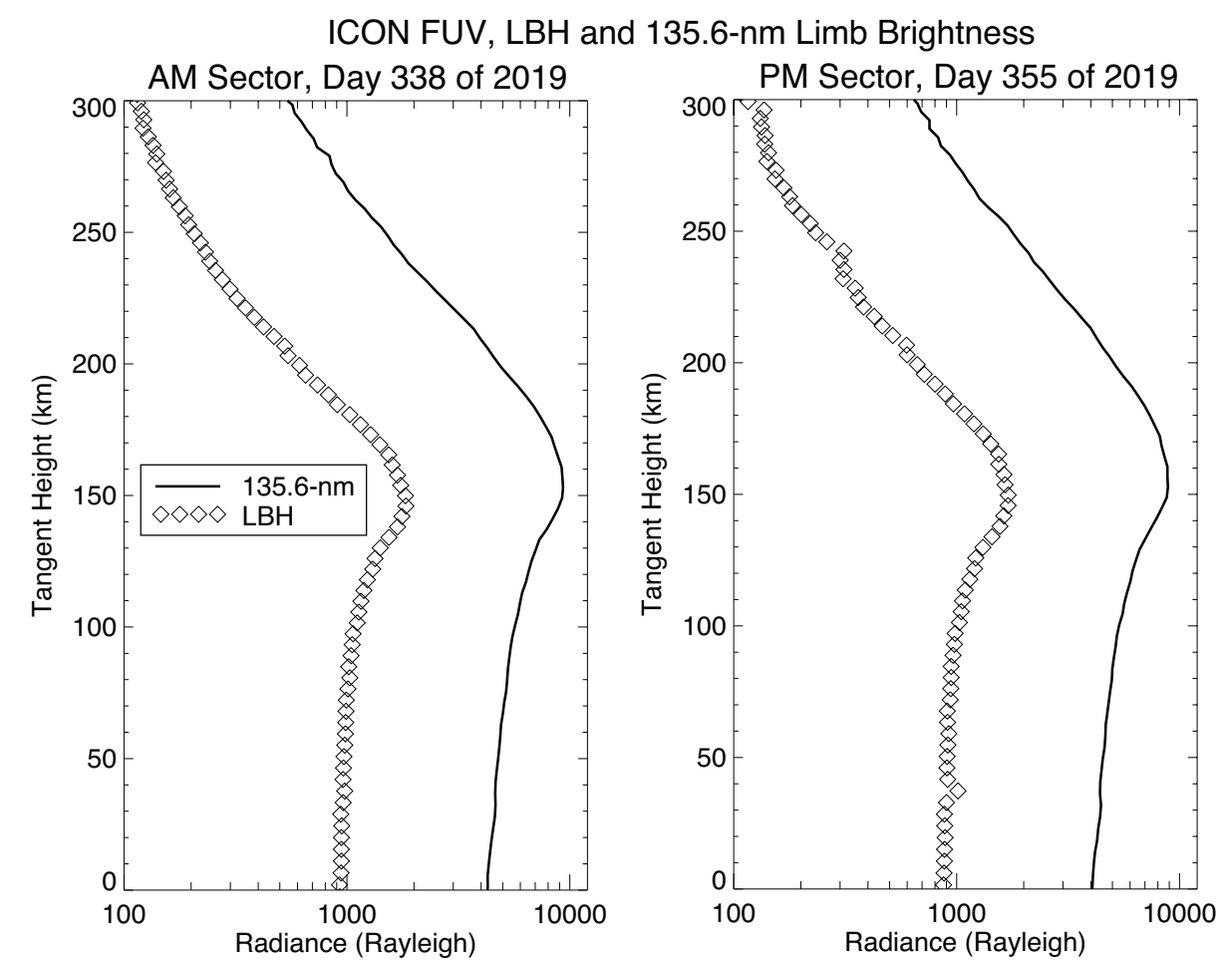

Figure 5. Altitude profiles in AM and PM sectors from ICON FUV for OI and $\mathrm{N}_{2}$ emissions at 135.6-nm and in LBH bands. The GOLD AM sector is observed by ICON on Day 338, 2019, while the PM sector is observed on Day 355. Solid lines show the 135.6-nm emission intensity and open symbols show that of LBH.

\section{Conclusions}

The GOLD observations provide a unique new view of the daytime thermosphere, allowing for new research efforts to understand the variability of the thermosphere-ionosphere system. GOLD observes that the emissions of thermospheric atomic oxygen vary from one day to the next in a way that differs signficantly from the closely related thermospheric $\mathrm{N}_{2}$ emissions. The LBH emission exhibits changes that are well correlated across the dayside, while the OI emission is not. This indicates a solar driver as the primary cause for LBH emissions, with another source adding to the OI variation. With ICON providing evidence that the OI and LBH emissions originate from nearly the same altitudes in the thermosphere around $140-150 \mathrm{~km}$, and the OI and LBH emissions in the same regions show practically no correlation, we conclude that the oxygen densities themselves at this altitude range are varying in a manner that is unconnected to the nitrogen density. Because of the general use of these emissions in retrieving ratios of oxygen to nitrogen in the thermosphere, we submit that changes in this ratio are largely driven by changes in $\mathrm{OI}$, with $\mathrm{N}_{2}$ providing a slowly reference for comparison.

These types of observations direct new interest in understanding the production and loss of OI in the mesosphere and lower thermosphere, and the day-to-day changes in temperatures at the mesopause that themselves would drive temperature and density changes at higher altitudes. The GOLD mission, with its capability of retrieving effective temperatures in the region of the $\mathrm{LBH}$ emissions, could further contribute to the investigation with daily retrievals of temperatures in these regions for comparison to the changes in OI brightness.

Author Contributions: Conceptualization, T.J.I.; methodology, T.J.I.; software, T.J.I.; analysis, T.J.I.; investigation, T.J.I.; resources, T.J.I.; data curation, R.W.E., W.E.M., S.B.M., H.U.F., S.L.E., C.T.; writing—original draft preparation, T.J.I., R.W.E.; visualization, T.J.I. All authors have read and agreed to the published version of the manuscript.

Funding: R.E. and W.M. are supported by NASA's Explorers Program through contract 80GSFC18C006. T.I., S.M., H.F., C.T. and S.E. are supported by NASA's Explorers Program through contract NNG12FA45C. 
Acknowledgments: We would like to thank the GOLD and ICON teams for the excellent data products, and the contributions of Robert Meier and Andrew Stephan to developing the Level 1 ICON FUV product. NASA GOLD data are processed in the GOLD science data center (https: / / gold.cs.ucf.edu) and downloaded from the NASA Space Physics Data Archive (https:/ / spdf.gsfc.nasa.gov). ICON data are processed in the ICON data center and available in June 2020 from the ICON science data center (https:/ /icon.ssl.berkeley.edu).

Conflicts of Interest: The authors declare that no conflicts of interest exist in the reporting of these research results.

\section{Abbreviations}

The following abbreviations are used in this manuscript:

F107 solar 10.7-cm radio flux

LBH Lyman Birge Hopfield

\section{References}

1. $\quad$ Eastes, R.W.; McClintock, W.E.; Burns, A.G.; Anderson, D.N.; Andersson, L.; Codrescu, M.; Correira, J.T.; Daniell, R.E.; England, S.L.; Evans, J.S.; et al. The Global-Scale Observations of the Limb and Disk (GOLD) Mission. Space Sci. Rev. 2017. [CrossRef]

2. $\quad$ Eastes, R.W.; McClintock, W.E.; Burns, A.G.; Anderson, D.N.; Andersson, L.; Aryal, S.; Budzien, S.A.; Cai, X.; Codrescu, M.V.; Correira, J.T.; et al. Initial Observations by the GOLD Mission. J. Geophys. Res. 2020, 125, e2020JA027823. [CrossRef]

3. Mlynczak, M.; Martin-Torres, F.J.; Russell, J.; Beaumont, K.; Jacobson, S.; Kozyra, J.; Lopez-Puertas, M.; Funke, B.; Mertens, C.; Gordley, L.; et al. The natural thermostat of nitric oxide emission at $5.3 \mu \mathrm{m}$ in the thermosphere observed during the solar storms of April 2002. Geophys. Res. Lett. 2003, 30, 2100. [CrossRef]

4. Meier, R.R. Ultraviolet Spectroscopy and Remote Sensing of the Upper Atmosphere. Space Sci. Rev. 1991, 58, 1-185. [CrossRef]

5. Hanson, W.B. Radiative recombination of atomic oxygen ions in the nighttime F-region. J. Geophys. Res. 1969, 74, 3720-3722. [CrossRef]

6. Hicks, G.T.; Chubb, T.A. Equatorial aurora/airglow in the far ultraviolet. J. Geophys. Res. 1970, 75, $6233-6248$. [CrossRef]

7. Carruthers, G.R.; Page, T. Apollo 16 far ultraviolet imagery of the polar auroras, tropical airglow belts, and general airglow. J. Geophys. Res. 1976, 81, 483-496. [CrossRef]

8. Meier, R.R.; Prinz, D.K. Observations of the O I 1304-A airglow from Ogo 4. J. Geophys. Res. 1971, 76, 4608-4620. [CrossRef]

9. Conway, R.R.; Meier, R.R.; Huffman, R.E. Satellite observations of the OI 1304, 1356 and 1641 Ådayglow and the abundance of atomic oxygen in the thermosphere. Planet. Space Sci. 1988, 36, 963-973. [CrossRef]

10. Craven, J.D.; Nicholas, A.C.; Frank, L.A.; Strickland, D.J.; Immel, T.J. Variations in FUV dayglow with intense auroral activity. Geophys. Res. Lett. 1994, 21, 2793-2796. [CrossRef]

11. Immel, T.J.; Craven, J.D.; Nicholas, A.C. The DE-1 auroral imager's response to the FUV dayglow for thermospheric studies. J. Atmos. Sol.-Terr. Phys. 2000, 62, 47-64. [CrossRef]

12. Frank, L.A.; Craven, J.D.; Burch, J.L.; Winningham, J.D. Polar views of the Earth's aurora with Dynamics Explorer. Geophys. Res. Lett. 1982, 9, 1001-1004. [CrossRef]

13. Craven, J.D.; Frank, L.A. Latitudinal motions of the aurora during substorms. J. Geophys. Res. 1987, 92, $4565-4573$. [CrossRef]

14. Killeen, T.L.; Craven, J.D.; Frank, L.A.; Ponthieu, J.J.; Spencer, N.W.; Heelis, R.A.; Brace, L.H.; Roble, R.G.; Hays, P.B.; Carignan, G.R. On the relationship between dynamics of the polar thermosphere and morphology of the aurora: Global-scale observations from Dynamics Explorer 1 and 2. J. Geophys. Res. 1988, 93, 2675-2692. [CrossRef]

15. Immel, T.J.; Eastes, R.W. New NASA Missions Focus on Terrestrial Forcing of the Space Environment. Bull. Am. Meteo. Soc. 2019, 100, 2153-2156. [CrossRef]

16. Krywonos, A.; Murray, D.J.; Eastes, R.W.; Aksnes, A.; Budzien, S.A.; Daniell, R.E. Remote sensing of neutral temperatures in the Earth's thermosphere using the Lyman-Birge-Hopfield bands of $\mathrm{N}_{2}$ : Comparisons with satellite drag data. J. Geophys. Res. 2012, 117, A09311. [CrossRef] 
17. Ajello, J.M.; Shemansky, D.E. A reexamination of important $\mathrm{N}_{2}$ cross sections by electron impact with application to the dayglow: The Lyman-Birge-Hopfield band system and N I(119.99 nm). J. Geophys. Res. 1985, 90, 9845-9861. [CrossRef]

18. Ajello, J.M.; Malone, C.P.; Holsclaw, G.M.; Hoskins, A.C.; Eastes, R.W.; McClintock, W.E.; Johnson, P.V. Electron impact study of the $100 \mathrm{eV}$ emission cross section and lifetime of the Lyman-Birge-Hopfield band system of $\mathrm{N}_{2}$ : Direct excitation and cascade. J. Geophys. Res. 2017, 122, 6776-6790. [CrossRef]

19. Strickland, D.J.; Evans, J.S.; Paxton, L.J. Satellite remote sensing of thermospheric O/N2 and solar EUV. 1: Theory. J. Geophys. Res. 1995, 100, 12217-12226. [CrossRef]

20. Evans, J.S.; Strickland, D.J.; Huffman, R.E. Satellite remote sensing of thermospheric O/N2 and solar EUV. 2: Data analysis. J. Geophys. Res. 1995, 100, 12227-12233. [CrossRef]

21. Zhang, Y.; Paxton, L.J.; Morrison, D.; Wolven, B.; Kil, H.; Meng, C.I.; Mende, S.B.; Immel, T.J. O/ $\mathrm{N}_{2}$ changes during 1-4 October 2002 storms: IMAGE SI-13 and TIMED/GUVI observations. J. Geophys. Res. 2004, 109, 10308. [CrossRef]

22. Meier, R.R.; Crowley, G.; Strickland, D.J.; Christensen, A.B.; Paxton, L.J.; Morrison, D.; Hackert, C.L. First look at the 20 November 2003 superstorm with TIMED/GUVI: Comparisons with a thermospheric global circulation model. J. Geophys. Res. 2005, 110. [CrossRef]

23. Zhang, Y.; Paxton, L.J. Long-term variation in the thermosphere: TIMED/GUVI observations. J. Geophys. Res. 2011, 116, A00H02. [CrossRef]

24. Zhang, Y.; Paxton, L.J. Reply to comment by D.J. Strickland et al. on "Long-term variation in the thermosphere: TIMED/GUVI observations". J. Geophys. Res. 2012, 117. [CrossRef]

25. Strickland, D.J.; Evans, J.S.; Correira, J. Comment on "Long-term variation in the thermosphere: TIMED/GUVI observations" by Y. Zhang and L. J. Paxton. J. Geophys. Res. 2012, 117. [CrossRef]

26. McClintock, W.E.; Eastes, R.W.; Hoskins, A.C.; Siegmund, O.H.W.; McPhate, J.B.; Krywonos, A.; Solomon, S.C.; Burns, A.G. Global-Scale Observations of the Limb and Disk Mission Implementation: 1. Instrument Design and Early Flight Performance. J. Geophys. Res. 2020, 125, e2020JA027797, [CrossRef]

27. Chakrabarti, S.; Paresce, F.; Bowyer, S.; Kimble, R.; Kumar, S. The extreme ultraviolet day airglow. J. Geophys. Res. 1983, 88, 4898-4904. [CrossRef]

28. Correira, J.; Evans, J.S.; Viebell, V.; Eastes, R. GOLD Level 2 Data Products: O/ $\mathrm{N}_{2}$ and QEUV Algorithms. J. Geophys. Res. 2020, submitted.

29. Gan, Q.; Eastes, R.W.; Burns, A.G.; Wang, W.; Qian, L.; Solomon, S.C.; Codrescu, M.V.; McInerney, J.; McClintock, W.E. First Synoptic Observations of Geomagnetic Storm Effects on the Global-Scale OI 135.6-nm Dayglow in the Thermosphere by the GOLD Mission. Geophys. Res. Lett. 2020, 47, e2019GL085400, [CrossRef]

30. Eastes, R.W.; Solomon, S.C.; Daniell, R.E.; Anderson, D.N.; Burns, A.G.; England, S.L.; Martinis, C.R.; McClintock, W.E. Global-Scale Observations of the Equatorial Ionization Anomaly. Geophys. Res. Lett. 2019, 46, 9318-9326. [CrossRef]

31. Kamalabadi, F.; Qin, J.; Harding, B.J.; Iliou, D.; Makela, J.J.; Meier, R.R.; England, S.L.; Frey, H.U.; Mende, S.B.; Immel, T.J. Inferring Nighttime Ionospheric Parameters with the Far Ultraviolet Imager Onboard the Ionospheric Connection Explorer. Space Sci. Rev. 2018, 214, 70. [CrossRef]

32. Immel, T.J.; England, S.L.; Mende, S.B.; Heelis, R.A.; Englert, C.R.; Edelstein, J.; Frey, H.U.; Korpela, E.J.; Taylor, E.R.; Craig, W.W.; et al. The Ionospheric Connection Explorer Mission: Mission Goals and Design. Space Sci. Rev. 2018, 214, 13. [CrossRef]

33. Stephan, A.W.; Meier, R.R.; England, S.L.; Mende, S.B.; Frey, H.U.; Immel, T.J. Daytime O/N 2 Retrieval Algorithm for the Ionospheric Connection Explorer (ICON). Space Sci. Rev. 2018, 214, 42. [CrossRef]

34. Rostoker, G. Geomagnetic indices. Rev. Geophys. 1972, 10, 935-950. [CrossRef]

35. Tapping, K.F. The 10.7-cm solar radio flux (F10.7). Space Weather 2013, 11, 394-406. [CrossRef]

36. Prölss, G.W.; Roemer, M. Thermospheric Storms. Adv. Space Res. 1987, 7, 223-235. [CrossRef]

37. Burns, A.G.; Killeen, T.L.; Carignan, G.R.; Roble, R.G. Large enhancements of the $\mathrm{O} / \mathrm{N}_{2}$ ratio in the evening sector of the winter hemisphere during geomagnetic storms. J. Geophys. Res. 1995, 100, 14673-14691. [CrossRef]

38. Immel, T.J.; Crowley, G.; Craven, J.D.; Roble, R.G. Dayside enhancements of thermospheric O/ $\mathrm{N}_{2}$ following magnetic storm onset. J. Geophys. Res. 2001, 106, 15471-15488. [CrossRef]

39. Fuller-Rowell, T.J.; Codrescu, M.V.; Moffett, R.J.; Quegan, S. Response of the thermosphere and ionosphere to geomagnetic storms. J. Geophys. Res. 1994, 99, 3893-3914. [CrossRef] 
40. Rishbeth, H.; Heelis, R.; Müller-Wodarg, I. Variations of thermospheric composition according to AE-C data and CTIP modelling. Ann. Geophys. 2004, 22, 441-452. [CrossRef]

41. Mende, S.B.; Frey, H.U.; Rider, K.; Chou, C.; Harris, S.E.; Siegmund, O.H.W.; England, S.L.; Wilkins, C.; Craig, W.; Immel, T.J.; et al. The Far Ultra-Violet Imager on the Icon Mission. Space Sci. Rev. 2017, 212, 655-696. [CrossRef]

42. Gardner, L.C.; Schunk, R.W. Large-scale gravity wave characteristics simulated with a high-resolution global thermosphere-ionosphere model. J. Geophys. Res. 2011, 116, A06303. [CrossRef]

43. Nishioka, M.; Tsugawa, T.; Kubota, M.; Ishii, M. Concentric waves and short-period oscillations observed in the ionosphere after the 2013 Moore EF5 tornado. Geophys. Res. Lett. 2013, 40, 5581-5586. [CrossRef]

44. England, S.L.; Greer, K.R.; Solomon, S.C.; Eastes, R.W.; McClintock, W.E.; Burns, A.G. Observation of Thermospheric Gravity Waves in the Southern Hemisphere With GOLD. J. Geophys. Res. 2020, 125, e27405. [CrossRef]

45. Hagan, M.E.; Forbes, J.M. Migrating and nonmigrating semidiurnal tides in the upper atmosphere excited by tropospheric latent heat release. J. Geophys. Res. 2003, 108. [CrossRef]

46. Liu, H.L.; Yudin, V.A.; Roble, R.G. Day-to-day ionospheric variability due to lower atmosphere perturbations. Geophys. Res. Lett. 2013, 40, 665-670. [CrossRef]

47. Pedatella, N.M.; Oberheide, J.; Sutton, E.K.; Liu, H.L.; Anderson, J.L.; Raeder, K. Short-term nonmigrating tide variability in the mesosphere, thermosphere, and ionosphere. J. Geophys. Res. 2016, 121, 3621-3633. [CrossRef]

48. Mlynczak, M.G.; Solomon, S. A detailed evaluation of the heating efficiency in the middle atmosphere. J. Geophys. Res. 1993, 98, 10517-10541. [CrossRef]

49. Mlynczak, M.G.; Hunt, L.A.; Mast, J.C.; Thomas Marshall, B.; Russell, J.M., IIII Smith, A.K.; Siskind, D.E.; Yee, J.H.; Mertens, C.J.; Javier Martin-Torres, F.; et al. Atomic oxygen in the mesosphere and lower thermosphere derived from SABER: Algorithm theoretical basis and measurement uncertainty. J. Geophys. Res. 2013, 118, 5724-5735. [CrossRef]

(C) 2020 by the authors. Licensee MDPI, Basel, Switzerland. This article is an open access article distributed under the terms and conditions of the Creative Commons Attribution (CC BY) license (http:/ / creativecommons.org/licenses/by/4.0/). 